Check for updates

Cite this: Mater. Chem. Front. 2020, 4, 1411

Received 5th December 2019 , Accepted 15th February 2020

DOI: $10.1039 / \mathrm{c} 9 q m 00737 g$

rsc.li/frontiers-materials

\title{
The origin of the unusual red-shifted aggregation- state emission of triphenylamine-imidazole molecules: excimers or a photochemical reaction?†
}

\author{
Jin Liu, ${ }^{a}$ Jianai Chen, ${ }^{a}$ Yujie Dong, ${ }^{a}$ Yue Yu, ${ }^{a}$ Shitong Zhang, ${ }^{b}$ Jianli Wang, (D) ${ }^{a}$ \\ Qingbao Song, ${ }^{a}$ Weijun Li (D) *a and Cheng Zhang (D)*a
}

\begin{abstract}
At the moment, there is still no consensus regarding the origin of the newly apparent unusual red-shifted aggregation-state emission arising from some triphenylamine-imidazole luminescent materials. One view is that the unusual emission originates from the formation of excimers, but a recently observed photochemical reaction also seems to contribute to it. Herein, a new imidazole molecule, TPDBM, was synthesized to investigate the actual origin of this phenomenon. Compared with a dilute solution of TPDBM, its film obviously exhibits newly apparent high red-shifted emission at around $520 \mathrm{~nm}$ in the PL spectrum. According to UV-vis spectra and aggregation-induced emission (AIE) and concentrationdependent emission results, it seems that the red-shifted emission is derived from excimers. However, further studies of excitation spectra, in situ PL spectra and oxygen-free PL spectra entirely overturn this conclusion and ultimately confirm that it seems to come from a photochemical reaction occurring on the imidazole ring of TPDBM. Two photochemical products, TPBMZ and TPDYZ, with excellent AIE effects were obtained through this photochemical reaction, and TPBMZ can be further oxidized to TPDYZ due to the quite fast reaction rate. Furthermore, the photochemical reaction of TPDBM doped into PMMA film was suitably applied as a fluorescent probe for oxygen with a lowest detectable oxygen volume ratio of $0.1 \%$.
\end{abstract}

\section{Introduction}

As a classical molecular structural unit of functional materials, ${ }^{1-3}$ imidazole possesses a typical five-member ring containing two entirely different nitrogen atoms, where one is electron-rich and the other is electron-deficient, which allows great control of the photoelectrical properties of imidazole derivatives. This is why imidazole derivatives can be extensively applied to OLEDs, ${ }^{4-12}$ fluorescent switches, ${ }^{13-15}$ optical sensors, ${ }^{16-18}$ electron transfer mediators, ${ }^{19}$ memory devices ${ }^{20}$ and other fields of interest. ${ }^{21-26}$ With the requirement for improved material performance, it is even more necessary to further explore imidazole molecules with new structures and investigate their photophysical and photochemical properties.

\footnotetext{
${ }^{a}$ State Key Laboratory Breeding Base of Green Chemistry-Synthesis Technology, College of Chemical Engineering, Zhejiang University of Technology, Hangzhou, 310014, P. R. China.E-mail: liwj@zjut.edu.cn, czhang@zjut.edu.cn

${ }^{b}$ State Key Lab of Supramolecular Structure and Materials, Jilin University, Changchun 130012, P. R. China

$\dagger$ CCDC 1969967. For crystallographic data in CIF or other electronic format see DOI: $10.1039 / \mathrm{c} 9 \mathrm{qm} 00737 \mathrm{~g}$
}

Triphenylamine, as a well-known electron donor, ${ }^{27-32}$ has been introduced to build imidazole molecules ${ }^{33-36}$ that have shown excellent photoelectrical properties, such as high quantum efficiencies, ${ }^{35}$ balanced charge mobilities, ${ }^{36}$ and so on. Many interesting and impressive studies have been already reported describing the luminescence properties of triphenylamineimidazole molecules. ${ }^{37-41}$ For example, Li et al. developed a molecule with deep-blue emission, TPA-PIM, with an extraordinarily narrow FHWM of $35 \mathrm{~nm} .{ }^{38}$ And a similar molecule (PTN) was reported by Du et al. with newly apparent unusual red-shifted emission in the aggregation state, which was attributed to excimer formation by the authors. ${ }^{39}$ Recently, a photochemical reaction on the imidazole ring of this kind of molecule (DPA-PIM) was reported, ${ }^{40}$ and the photochemical products also displayed high red-shifted emission in the aggregation state with yellow fluorescence under UV and air conditions. Based on these studies, it is necessary to undertake deep and careful investigations to further confirm whether this kind of unusual emission from imidazole molecules is due to excimers or a photochemical reaction.

Herein, we designed and successfully synthesized a new triphenylamine-imidazole molecule, TPDBM, with two imidazole rings linked on one triphenylamine unit. An obvious large red-shifted 
emission peak at around $520 \mathrm{~nm}$ appeared in the PL spectrum of TPDBM film compared to in solution. We discussed the source of this unusual emission based on spectroscopic analysis and investigations of photochemical reactions involving TPDBM, and finally arrived at the conclusion that this unusual red-shifted emission comes from a photochemical reaction occurring on the imidazole ring of TPDBM and not from excimer formation. Furthermore, two photochemical products, TPBMZ and TPDYZ, were obtained through this photochemical reaction, and TPBMZ could be further turned to TPDYZ under the same conditions due to the quite fast reaction rate. Surprisingly, both of the photochemical products exhibited excellent aggregation-induced emission (AIE) properties. Moreover, the photochemical reaction involving TPDBM doped into PMMA film was applied to a fluorescent probe for oxygen, which displayed excellent performance with a lowest detectable oxygen volume ratio of $0.1 \%$.

\section{Results and discussion}

\section{Material synthesis and characterization}

As shown in Fig. 1, the triphenylamine-imidazole molecule TPDBM was prepared simply via a cyclization reaction ${ }^{40}$ from benzil, aniline, ammonium acetate and the intermediate compound 4,4'-(phenylazanediyl)dibenzaldehyde (M1 in Fig. 1), which was newly synthesized via an improved process with a yield of $80 \%$ according to the related ref. 42 . The structure of the target product TPDBM was confirmed via $^{1} \mathrm{H}$ NMR and mass spectroscopy studies. All the reaction details relating to this work can be found in the Experimental section.

\section{Photophysical properties}

UV-vis and PL spectroscopy studies were carried out to investigate the basic photophysical properties of TPDBM. As shown in Fig. 2, when TPDBM was excited, significantly different PL spectra were obtained from TPDBM in solution and as a film. A diluted toluene solution of TPDBM $\left(10^{-5} \mathrm{M}\right)$ exhibited a relatively narrow single emission peak at around $405 \mathrm{~nm}$. But as a film, except for the normal emission peak at around $430 \mathrm{~nm}$, another large red-shifted emission peak at around $520 \mathrm{~nm}$ was also observed. Furthermore, when the above-mentioned TPDBM film possessing dual-emission properties was re-dissolved in toluene, the emission at around $520 \mathrm{~nm}$ disappeared and the spectrum became identical to the previous regular toluene solution. This transformation process can be well observed from the fluorescence images shown in the inset of Fig. 2. Also, the UV-vis spectra of TPDBM both in toluene and as

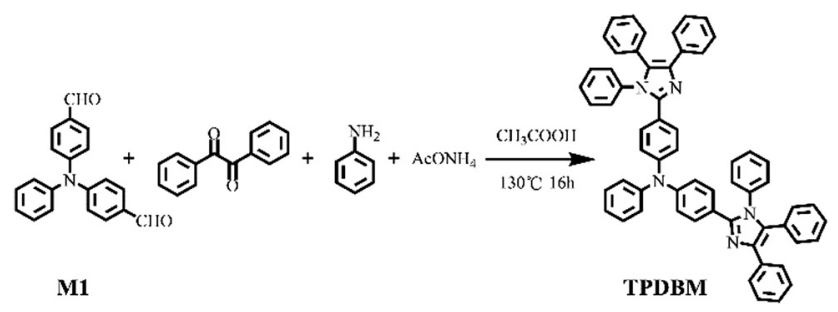

Fig. 1 The molecular structure and synthesis route of TPDBM.

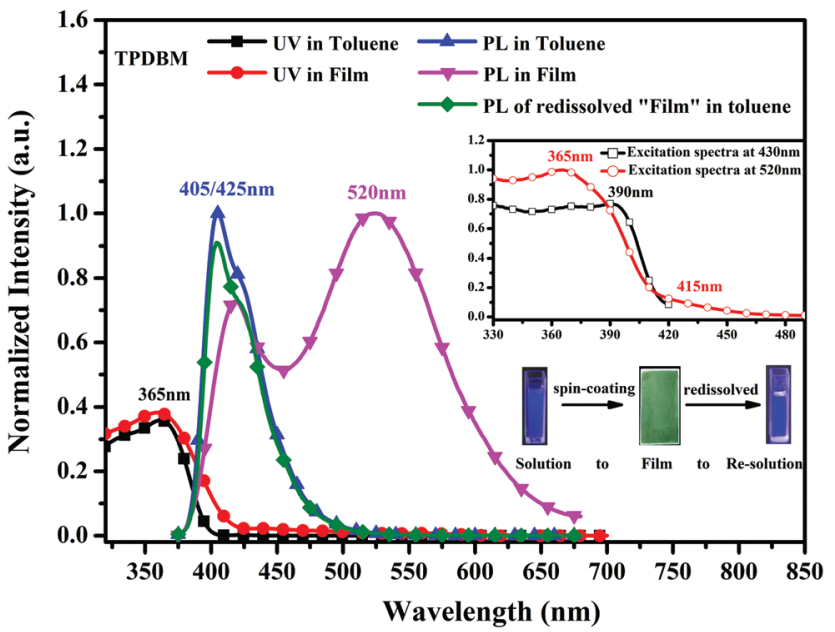

Fig. 2 UV-vis and PL spectra of TPDBM in toluene solution and film states; the inset shows the excitation spectra of TPDBM film at $430 \mathrm{~nm}$ and $520 \mathrm{~nm}$.

a film exhibited almost identical broad absorption bands located at around $365 \mathrm{~nm}$, indicating that the emission of TPDBM seems to originate from the same ground state whether in a solution or film state. Thus, this unusual large red-shifted emission peak of TPDBM in the film state seems to be related to the molecular aggregation state.

To further investigate the influence of the degree of molecular aggregation on the formation of this large red-shifted emission peak, PL spectra of TPDBM in THF and $\mathrm{H}_{2} \mathrm{O}$ mixed solutions with different water fraction $\left(f_{\mathrm{w}}\right)$ levels were measured (Fig. 3a). As TPDBM is almost insoluble in $\mathrm{H}_{2} \mathrm{O}$, it aggregates as the amount of $\mathrm{H}_{2} \mathrm{O}$ increases; therefore, the emission behaviour resulting from different aggregation degrees can provide clues for investigating the luminescence properties of TPDBM. TPDBM in dilute THF solution exhibited strong deep-blue emission at around $405 \mathrm{~nm}$ and its PL intensity remained nearly unchanged in the $\mathrm{THF} / \mathrm{H}_{2} \mathrm{O}$ mixture solution at a low water fraction. As the water fraction increased to $80 \%$, the emission peak at around $405 \mathrm{~nm}$ started to become weaker, whereas a new emission peak appeared at around $525 \mathrm{~nm}$ and further intensified as the water fraction was increased to $90 \%$. Meanwhile the fluorescence of the mixed solution changed from blue to green. This result indicated that a large degree of molecular aggregation could lead to the formation of red-shifted emission. Moreover, PL spectra of TPDBM in chloroform solutions of different molarity were also obtained (Fig. 3b). All the PL spectrum intensities were normalized for the convenient analysing of differences between the emission peaks. When TPDBM existed at a lower molarity $\left(\leq 10^{-4} \mathrm{M}\right)$, the PL spectrum exhibited only a single emission peak at around $412 \mathrm{~nm}$. As the molarity was increased to $5 \times 10^{-4} \mathrm{M}$ and $10^{-3} \mathrm{M}$, a new red-shifted emission peak appeared at around $530 \mathrm{~nm}$, which could also be described as resulting from an increase in the degree of molecular aggregation. So far, all the above-mentioned results demonstrated that the unusual large red-shifted emission peak was indeed related to a large degree of TPDBM molecular aggregation, which seems to be attributed to 

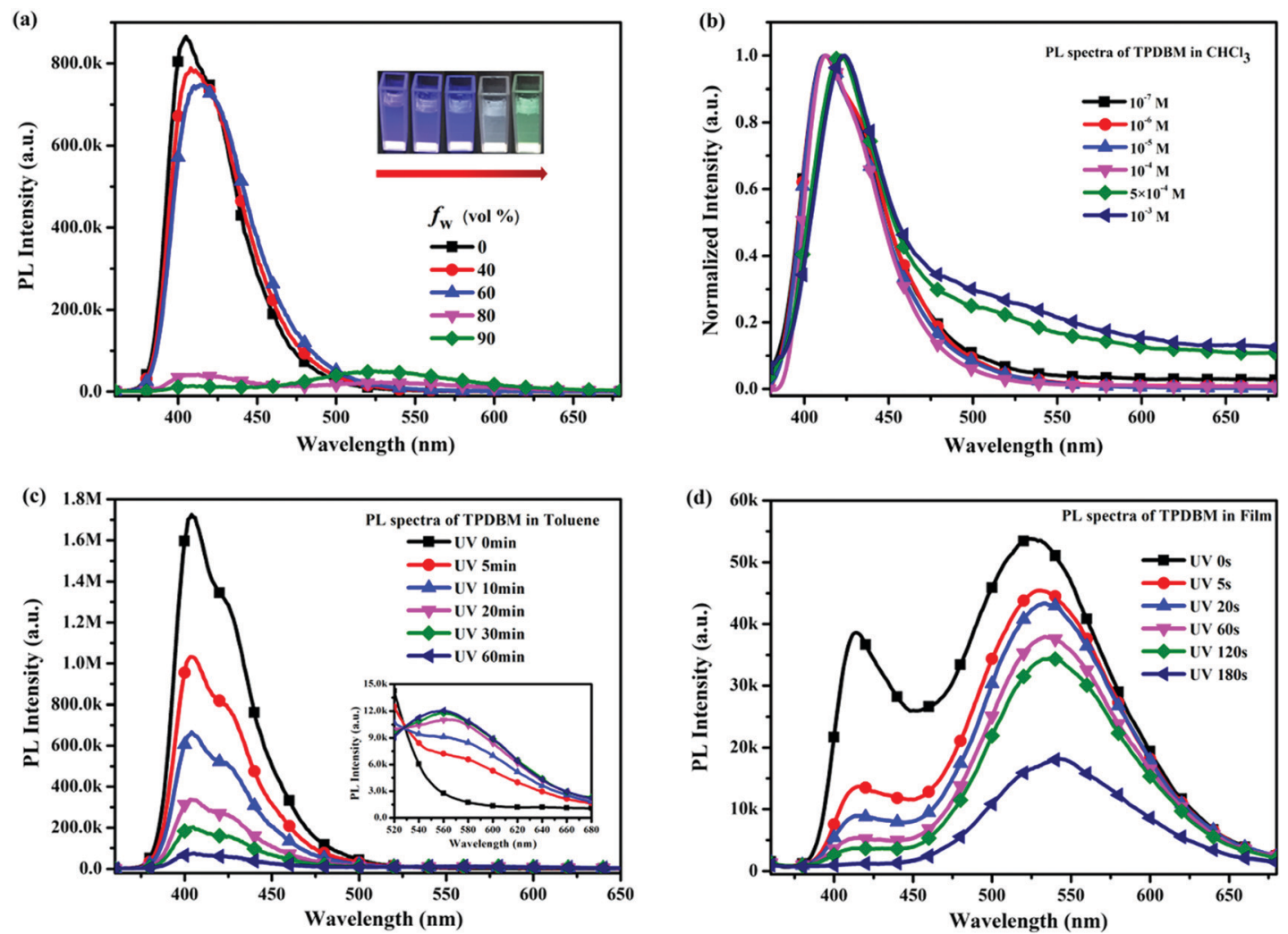

Fig. 3 (a) PL spectra of TPDBM in mixed solutions of THF and $\mathrm{H}_{2} \mathrm{O}$; the inset exhibits the actual luminescence of solutions with different water fractions. (b) PL spectra of TPDBM in chloroform solutions of different molarity. (c) In situ PL spectra of TPDBM in dilute toluene (10 $\left.{ }^{-5} \mathrm{M}\right)$ solutions under UV light $(365 \mathrm{~nm})$ for different periods of photo-irradiation; the emission wavelength spectra from 520 to $680 \mathrm{~nm}$ are magnified in the inset. (d) In situ PL spectra of TPDBM in film form.

the formation of excimers in the aggregated state and consistent with the report by Du et al.

However, as we looked at the excitation spectra of TPDBM films at 430 and $520 \mathrm{~nm}$ shown in Fig. 2, we were surprised to find that there was a marked difference between them, i.e., the former has only one peak at around $390 \mathrm{~nm}$, and the latter has two peaks at around 365 and $415 \mathrm{~nm}$. Unlike the UV spectra of TPDBM, this result indicates that the two emission peaks of TPDBM film come from different ground states, and the unusual large red-shifted emission peak from TPDBM film should not simply be described as arising from excimer emission. Further studies of $i n$ situ PL spectra of TPDBM in diluted toluene solution $\left(10^{-5} \mathrm{M}\right)$ also offered us new insights. As shown in Fig. 3c, as the photo-irradiation time increases, the PL intensity of the emission peak at around $405 \mathrm{~nm}$ gradually decreases; a small emission peak also appears at around $560 \mathrm{~nm}$ and becomes gradually enhanced. Since it is impossible to form excimers in dilute solutions, we could deduce that the red-shifted emission here could not arise from excimer emission but is more likely the result of photoinduced chemical changes following UV-irradiation. ${ }^{40}$ In addition, similar results were also obtained from in situ PL spectra of TPDBM in film form (Fig. 3d). As the irradiation time increased, the emission peak at around $420 \mathrm{~nm}$ kept getting weaker and almost completely disappeared after $180 \mathrm{~s}$. At the same time, the emission peak at around $520 \mathrm{~nm}$ was also gradually weakened at a relatively slow speed. These spectral changes indicated that TPDBM with strong deep-blue emission was constantly consumed during UV-irradiation and a new substance with relatively weak yellow emission was produced. These results clearly demonstrated that large levels of molecular aggregation are not the only necessary condition for the large red-shifted emission peak, and that a photochemical reaction involving TPDBM under UV-irradiation could also lead to this emission.

In order to further determine whether the actual origin of this large red-shifted emission peak of TPDBM in the film state relates to excimers or a photochemical reaction, PL spectra of TPDBM film in a pure $\mathrm{N}_{2}$ environment were measured. As shown in Fig. 4a, totally different to in air, the PL spectrum of TPDBM film in pure $\mathrm{N}_{2}$ exhibited only one emission peak at around $430 \mathrm{~nm}$. Moreover, when this film was returned to air, the large red-shifted emission peak at around $520 \mathrm{~nm}$ appeared again and the resulting PL spectrum exhibited identical dualemission to that measured originally in air. These results indicated that the presence of oxygen replaced the aggregation state as the indispensable condition for the generation of the unusual emission from the film; that is, the actual origin of this red-shifted emission is not likely to be excimer formation but is more likely to be the occurrence of a photochemical reaction. To further prove this point, we obtained three groups of in situ UV-vis spectra from TPDBM toluene solution (Fig. 4b). When the sample 
(a)

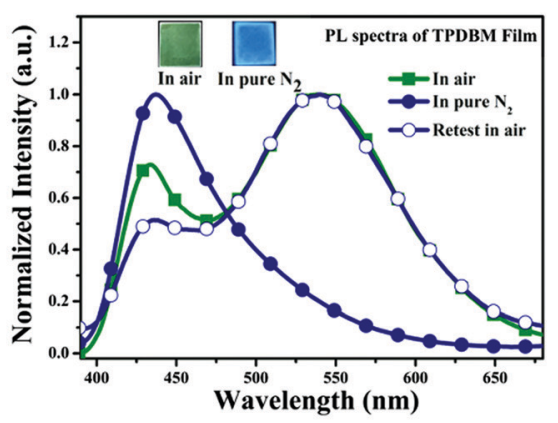

(b)

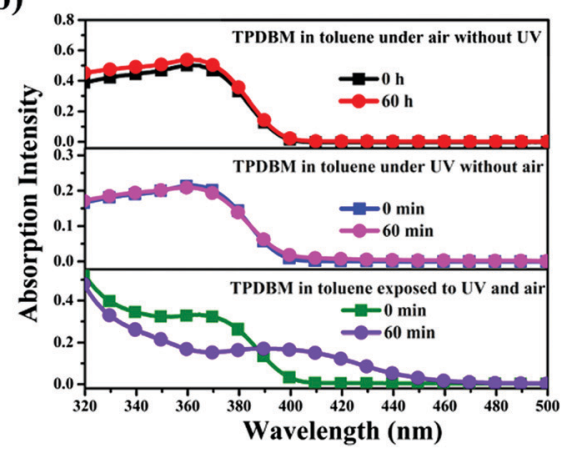

Fig. 4 (a) PL spectra of TPDBM film in air and in a pure $\mathrm{N}_{2}$ environment; the inset shows fluorescence images of TPDBM film in air and pure $\mathrm{N}_{2}$. (b) In situ UV spectra of TPDBM in toluene.

was kept in darkness without UV-irradiation or sealed in $\mathrm{N}_{2}$ without exposure to $\mathrm{O}_{2}$, the UV-vis spectra remained unchanged. But when it was simultaneously exposed to $\mathrm{UV}$ and $\mathrm{O}_{2}$ for $60 \mathrm{~min}$, the absorption changed obviously with a new peak appearing at around $410 \mathrm{~nm}$, which was consistent with the red-shifted emission seen in the excitation spectra. These results further supported the deduction that a photochemical reaction induced the red-shifted emission and pointed out that the two essential conditions for this photochemical reaction are the presence of UV-irradiation and oxygen from air.

\section{Photochemical reaction}

To identify how a photochemical reaction induced this unusual red-shifted emission, UV-irradiation experiments involving
TPDBM were carried out to obtain more information about this photochemical reaction and its products, as shown in Fig. 5. A high concentration of TPDBM dissolved in toluene could directly produce (Z)- $N$-((benzoylimino)(4-(phenyl(4-(1,4,5-triphenyl-1H-imidazol-2yl)phenyl)amino)phenyl)methyl)- $N$-phenylbenzamide (TPBMZ) in $40 \%$ yield and $N, N^{\prime}-\left(\left(1 Z, 1^{\prime} Z\right)-((\right.$ phenylazanediyl)bis(4,1-phenylene $))-$ bis((benzoylimino)methylene))bis( $N$-phenylbenzamide) (TPDYZ) in $20 \%$ yield under $365 \mathrm{~nm}$ UV-irradiation and in air (Fig. 5a); the molecular structures were confirmed via $^{1} \mathrm{H}$ NMR and mass spectroscopy studies. A single crystal of TPBMZ (CCDC number: $1969967 \dagger$ ) was obtained via the slow diffusion of $n$-hexane solvent into chloroform solution (Fig. 5b); this clearly shows the configuration, with one imidazole of TPDBM undergoing ring-opening, and also verifies that a photochemical reaction indeed occurred (a)

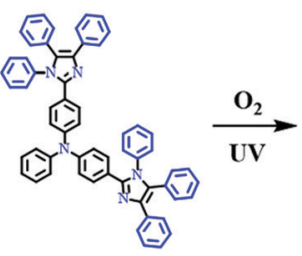

TPDBM

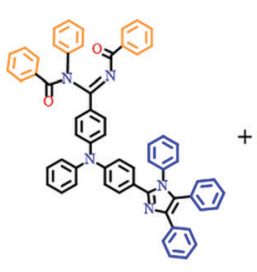

TPBMZ (c)

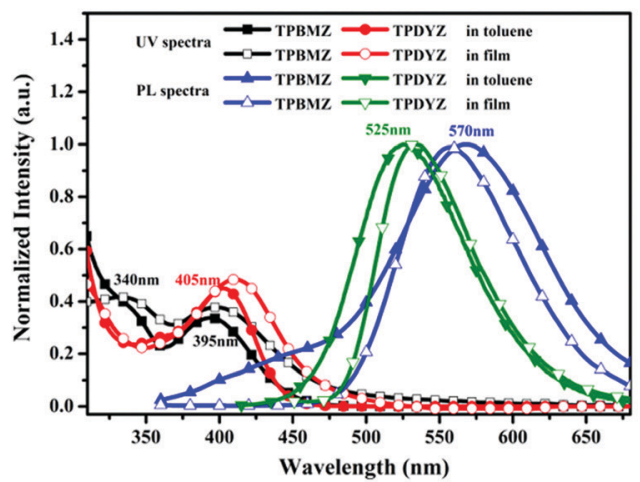

(b)

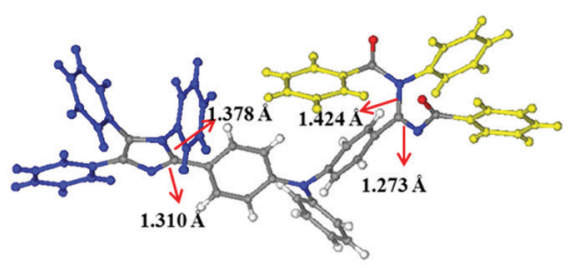

TPDYZ (d)

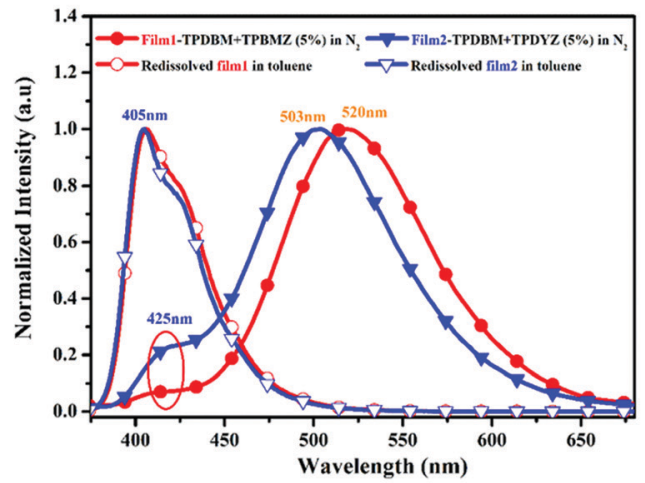

Fig. 5 (a) The photochemical reaction changing the molecular structure from TPDBM to TPBMZ and TPDYZ. (b) The single crystal structure of TPBMZ. (c) The UV and PL spectra of TPBMZ and TPDYZ both in toluene solution and film states. (d) The PL spectra of TPDBM doped with $5 \%$ (molarity) TPBMZ and TPDYZ in film form under pure $\mathrm{N}_{2}$ and in toluene solution under air. 
on the imidazole ring of TPDBM. Additionally, the structure of the TPBMZ crystal shows bond lengths of $1.27 \AA$ for $\mathrm{C}=\mathrm{N}$ and $1.42 \AA$ for $\mathrm{C}-\mathrm{N}$, effectively confirming that benzoylimino and benzamide structures were generated via the ring-opening reaction. $^{40}$

The basic photophysical properties of the two photochemical products were also studied using their UV and PL spectra (Fig. 5c). In the UV spectrum of TPBMZ dissolved in toluene, the absorption peak of TPDBM at around $365 \mathrm{~nm}$ blue-shifted to $340 \mathrm{~nm}$, while a new wide absorption band at around $395 \mathrm{~nm}$, corresponding to the ring-opening structure of the imidazole ring, was also observed. On account of the disappearance of the imidazole-ring structure, the other product, TPDYZ, showed only one absorption peak at around $405 \mathrm{~nm}$. Interestingly, the PL spectrum of TPBMZ in toluene exhibited dual emission, with two peaks at around 455 and $570 \mathrm{~nm}$, respectively, which was probably caused by the special molecular structure, while TPDYZ showed a single emission peak at around $525 \mathrm{~nm}$. The UV spectra of the two photochemical products in film form were very similar to those in toluene, with a slight red shift, and their PL spectra were virtually unchanged, except that the emission peak at around $455 \mathrm{~nm}$ from TPBMZ disappeared in the film state. The resulting UV and PL spectra from these two photochemical products were in very good agreement with the observed red-shifted emission and excitation spectra of TPDBM film.

Since the unusual red-shifted emission was indeed induced by a photochemical reaction involving the imidazole unit, why could it be observed obviously in the film and aggregation states but not in regular dilute solution? As shown in Fig. 5d, we prepared two types of TPDBM film doped with 5\% (molarity) TPBMZ and TPDYZ, respectively, and tested their luminescence behaviour. Similar to pure TPDBM film in air, and regardless of whether the TPDBM film was doped with TPBMZ or TPDYZ, their PL spectra under pure $\mathrm{N}_{2}$ both exhibited weak blue emission at around $425 \mathrm{~nm}$ and strong red-shifted emission located at around 520 or $503 \mathrm{~nm}$, respectively. This should be ascribed to efficient Förster resonance energy transfer in the films due to the high levels of overlap between the PL spectrum of TPDBM (405 nm, Fig. 2) and the absorption spectra of the two photochemical products (395 and 405 nm, Fig. 5c). Moreover, when these two films were re-dissolved in toluene in air, both of them exhibited only one emission peak at around $405 \mathrm{~nm}$, indicating that the impact of trace amounts of TPBMZ and TPDYZ dopants on the PL spectra of TPDBM in dilute solution is negligible. These results indicated that the unusual red-shifted emission of TPDBM in the aggregation state was actually emission from a small number of photochemical products excited by a Förster resonance energy transfer process, which is more effective in the aggregation state than in dilute solution. Therefore, although irradiation from the excitation light source unavoidably produced small amounts of photochemical products both in film and in toluene solution during the collection of PL spectra in air, the emission from TPDBM only exhibited obviously red-shifted emission peaks in film and not in toluene. This is why the emission peaks from TPDBM were so different between the toluene solution and film states, as shown in Fig. $3 \mathrm{c}$ and $\mathrm{d}$.
To further explore this photochemical reaction, in situ UV spectra of TPDBM in toluene were obtained to monitor the specific reaction process. As shown in Fig. 6a, as the photo-irradiation time increased, the absorption peak at around $365 \mathrm{~nm}$ was gradually weakened and blue-shifted to $340 \mathrm{~nm}$. Meanwhile, a new wide absorption peak at around $410 \mathrm{~nm}$ appeared and gradually increased in size. In addition, in situ UV spectra of TPDBM in the film state also exhibited a similar trend with a relatively faster reaction speed (Fig. 6b). These results clearly demonstrated that TPDBM gradually turned into the photochemical products TPBMZ and TPDYZ during this UV-irradiation process. The most noteworthy point is that there were no other special peaks apart from those of TPDBM and its photochemical products during this process, whether in toluene solution or film form, indicating that no by-products are generated. In addition, in situ ${ }^{1} \mathrm{H}$ NMR spectra of TPBMZ were also obtained to further describe this photochemical reaction process (Fig. 6c). The characteristic chemical shifts of pure TPBMZ and TPDYZ were distinctly demonstrated using their original ${ }^{1} \mathrm{H}$ NMR spectra in toluene- $\mathrm{d}_{8}$ solution. The ${ }^{1} \mathrm{H}$ NMR spectrum of TPBMZ was measured again after being exposed to UV light in air for $90 \mathrm{~min}$ and the peak intensities at 8.07 and 7.58 ppm were obviously decreased, which clearly showed that TPBMZ turned to TPDYZ during the UV-irradiation process. This powerfully demonstrated that TPBMZ as a photochemical reaction product could further turn to the other product TPDYZ under the same conditions. In addition, as we know, the photochemical reaction occurred on the imidazole ring structure. Further investigations into the natural transition orbital (NTO) distributions of TPBMZ showed that a suitable structure was located in the high-energy $\mathrm{S}_{3}$ excited state of TPBMZ (Fig. 6d). According to Kasha's rule, we inferred that the rate of this reaction is really very fast because it could occur even in the presence of very fast intersystem crossing processes from high-energy excited states to low-energy excited states.

\section{AIE phenomenon}

The AIE properties of the two photochemical products TPBMZ and TPDYZ were also investigated. Surprisingly, unlike TPDBM, both of the photochemical products exhibited obvious AIE phenomena, as revealed by the data and the photographs shown in Fig. 7. The PL spectrum of TPBMZ in pure THF showed weak dual emission with two peaks at $445 \mathrm{~nm}$ and $565 \mathrm{~nm}$ (Fig. 7a). In the $\mathrm{THF} / \mathrm{H}_{2} \mathrm{O}$ mixed solution system, as the water fraction increased, the PL peak intensity at $565 \mathrm{~nm}$ was greatly enhanced, accompanied by a slight blue shift to $560 \mathrm{~nm}$. Meanwhile, the PL peak intensity at $445 \mathrm{~nm}$ continuously decreased and even almost disappeared, which further indicated that this weak emission perhaps comes from the imidazole-ring structure without AIE activity located in the higher energy excited state. Similar AIE behaviour was also observed from TPDYZ (Fig. 7b), which further demonstrated that the AIE emission from the photochemical products could be attributed to the generation of benzoylimino and benzamide groups as a result of ring opening. In addition, the AIE properties of these two photochemical products further explained why there were no visible red-shifted emission peaks in the PL spectra of TPDBM in dilute toluene solution, despite the presence of small amounts of the photochemical products. 
(a)

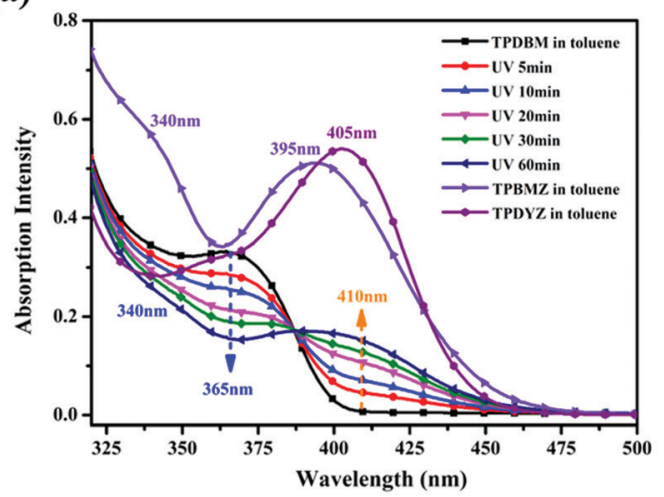

(c) The in situ 'H NMR spectra of TPBMZ in Toluene-d8

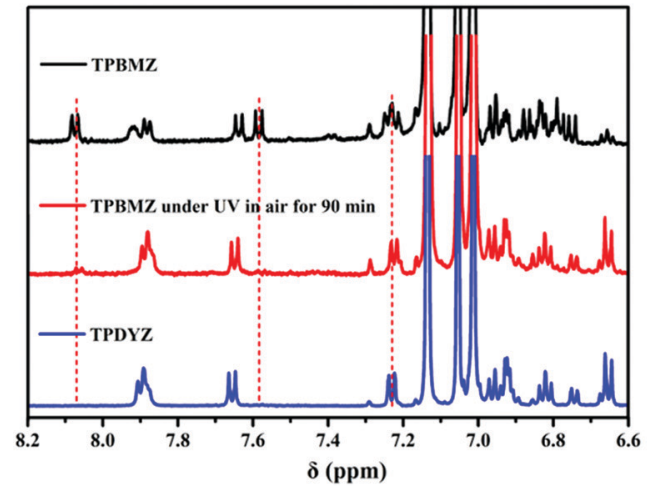

(b)

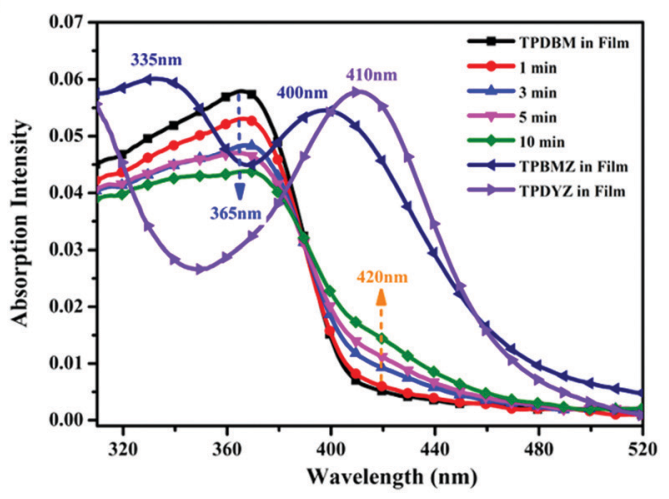

(d)

Vertical excited NTO

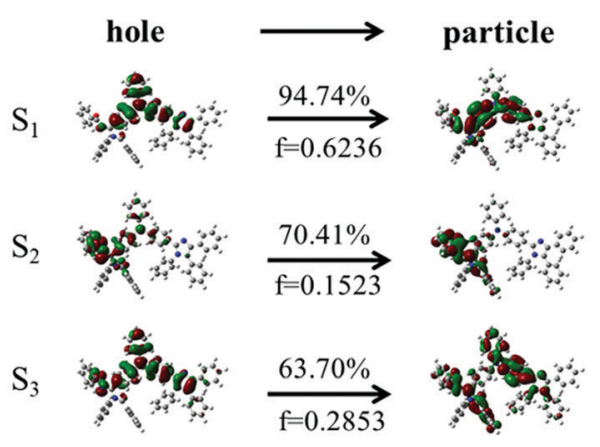

Fig. 6 In situ UV spectra of TPDBM (a) in toluene solution and (b) as a film. (c) In situ ${ }^{1} \mathrm{H}$ NMR spectra of TPBMZ. (d) The natural transition orbital (NTO) distributions of TPBMZ relating to $\mathrm{S}_{0} \rightarrow \mathrm{S}_{1}, \mathrm{~S}_{0} \rightarrow \mathrm{S}_{2}$ and $\mathrm{S}_{0} \rightarrow \mathrm{S}_{3}$ excitations at the TD-oB97X/6-31G(d,p) level using $\mathrm{S}_{0}$ state geometry.

\section{Oxygen detection}

Considering the outstanding sensitivity of TPDBM to oxygen and the quite rapid photochemical reaction rate, it could easily be applied to act as a fluorescent probe for oxygen. Furthermore, in order to meet the operational and low toxicity requirements for oxygen-sensing materials for practical applications, we doped TPDBM into PMMA film. ${ }^{43}$ The PL spectra of TPDBMdoped PMMA film in $\mathrm{N}_{2} / \mathrm{O}_{2}$ mixed gas with different volume ratios were investigated to roughly evaluate the oxygen sensitivity of this PMMA film. As shown in Fig. 8a, totally different PL spectra were obtained at different $\mathrm{O}_{2}$ concentrations. In comparison to the PL spectra in pure $\mathrm{N}_{2}$, although the $\mathrm{O}_{2}$ concentration was as low as $0.1 \%$, a slight but obvious change was still observed at around $500 \mathrm{~nm}$. Also, this emission gradually rose as the $\mathrm{O}_{2}$ concentration increased. This result was caused by the emergence of photochemical products in the PMMA film, according to the PL spectra of TPBMZ-doped PMMA film. Thus, the photochemical reaction of TPDBM-doped PMMA film could be applied as an efficient and hypotoxic fluorescent probe, with changes in the film fluorescence being used to detect oxygen. The lowest detectable oxygen volume ratio using this film was as low as $0.1 \%$, which was almost the best result compared to most other probes based on phosphorescence quenching theory. In addition, for the same reason, it is also easy to observe the UV-induced fluorescence tuning of TPDBM solid powder (Fig. 8b). When the blue-emitting TPDBM solid powder was ground under $365 \mathrm{~nm}$ UV-irradiation in air, its fluorescence rapidly turned to relatively weaker yellow fluorescence, while under pure $\mathrm{N}_{2}$ conditions, it remained unchanged. It is worth noting that this phenomenon was also reported by Anu Kundu et al., but they mistakenly thought that no chemical change occurred during this process. ${ }^{41}$ This demonstrates that TPDBM has great application potential in oxygen sensors and related photoelectrical areas as a result of the photochemical reaction.

\section{Experimental section}

\section{Materials and instruments}

All of the reagents and solvents were purchased from commercial sources and used directly without further purification. ${ }^{1} \mathrm{H}$ NMR spectra of the synthesized compounds were recorded using a Bruker AVANCE III instrument (Bruker, Switzerland). Mass spectra (MALDI-TOF-MS) analysis was performed using an AXIMACFRTM-plus instrument. Photophysical properties were investigated using a Shimadzu UV-1800 spectrophotometer (Shimadzu, Japan) and a HORIBA fluorolog-3 luminescence spectrophotometer (HORIBA, Japan). X-ray diffraction studies were carried out using an X'Pert Pro instrument and the single crystal structure was analysed using an Agilent Geminie instrument. Spin coating was carried out with a WS-650MZ-8NPPB spin coater. The UV 

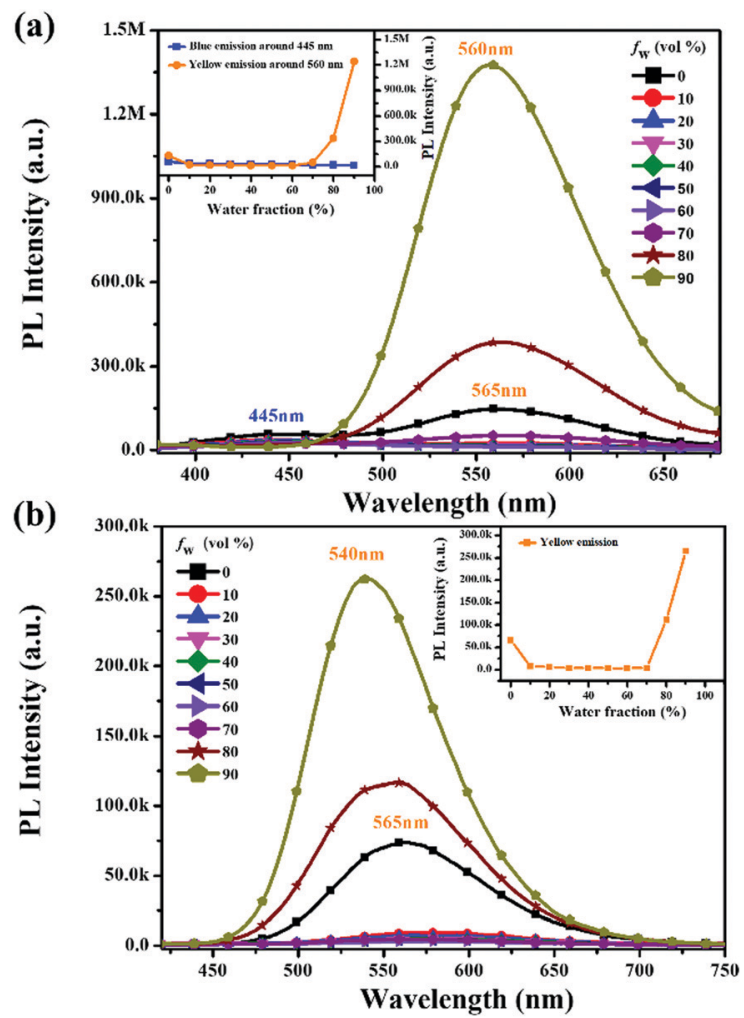

Fig. 7 The PL spectra of (a) TPBMZ and (b) TPDYZ in THF/ $\mathrm{H}_{2} \mathrm{O}$ mixed solution with different water fraction $\left(f_{\mathrm{w}}, \mathrm{v} / \mathrm{v}\right)$ levels; line charts showing the maximum PL peak intensities of TPBMZ and TPDYZ, respectively, are embedded in each part.

(a)

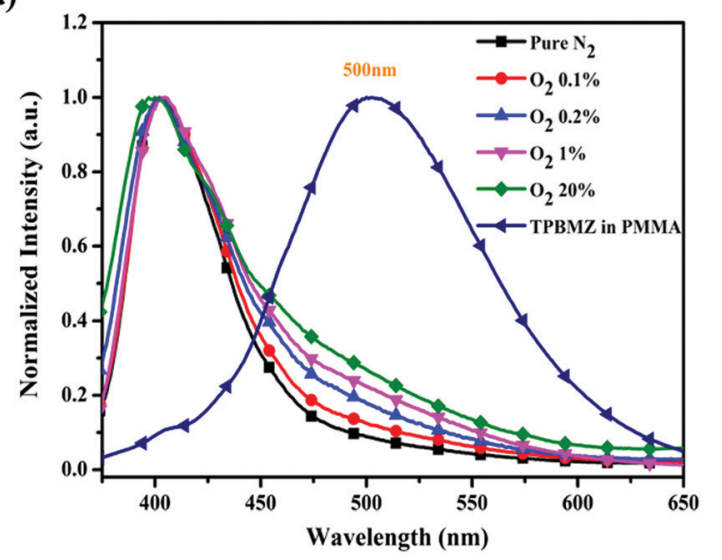

(b)

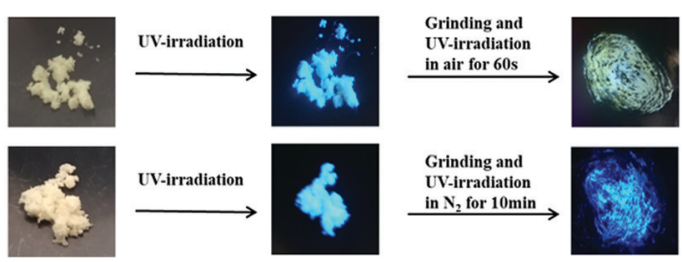

Fig. 8 (a) Normalized PL spectra of TPDBM doped PMMA film in $\mathrm{N}_{2} / \mathrm{O}_{2}$ mixed gas with different volume ratios. (b) Images of TPDBM solid powder under different treatment conditions. light used in the photochemical reaction was generated using a common ZF-7 portable UV light ( $6 \mathrm{~W}, 365 \mathrm{~nm})$.

For oxygen detection: the ratios of $\mathrm{N}_{2}$ and $\mathrm{O}_{2}$ were controlled, respectively, through adjusting gas flows using a gas flowmeter. $\mathrm{N}_{2}$ and $\mathrm{O}_{2}$ were thorough mixed until stable in a big container before measurements were taken. The speeds of the gas flows of $\mathrm{N}_{2}$ and $\mathrm{O}_{2}$ were controlled at 1000:1, 500:1, and 100:1 $\mathrm{ml} \mathrm{min}^{-1}$, respectively, to get ratios of about $0.1 \%, 0.2 \%$, and $1 \%$. The oxygen probe TPDBM was doped into PMMA film. PL spectrum measurements for oxygen detection were carried out using a HORIBA fluorolog-3 instrument; the excitation wavelength was $350 \mathrm{~nm}$, the emission wavelength range was $360 \mathrm{~nm}$ to $680 \mathrm{~nm}$, the slit width was $5 \mathrm{~nm}$, and the integration time was $1 \mathrm{~s}$. The irradiation all came from the excitation light source and, thus, the duration of irradiation for every measurement was about 320 seconds.

\section{Synthesis}

Synthesis of $4,4^{\prime}$-(phenylazanediyl)dibenzaldehyde (M1). In a round-bottom flask, $9.2 \mathrm{ml}$ of $\mathrm{POCl}_{3}$ was added to dry DMF $(11.2 \mathrm{ml})$ drop-by-drop at $0{ }^{\circ} \mathrm{C}$. After stirring for 45 minutes, triphenylamine $(1.96 \mathrm{~g}, 8 \mathrm{mmol})$ was added into the mixture and stirring was continued until the colour became yellow. Then, the reaction mixture was heated and stirred at $65{ }^{\circ} \mathrm{C}$ for $16 \mathrm{~h}$. After cooling to room temperature, the reaction mixture was poured into an ice-bath and neutralized with $\mathrm{NaOH}$ followed by extraction with ethyl acetate several times. The extracted organic phase was dried using anhydrous $\mathrm{MgSO}_{4}$ and filtered to obtain the crude product M1. Finally, M1 was further purified via column chromatography using petroleum ether/ethyl acetate $(10 / 1)$ as the eluent to afford a faint yellow solid powder $(1.9 \mathrm{~g}$, 80\%). ${ }^{1} \mathrm{H}$ NMR (500 MHz, DMSO, ppm): $\delta=9.89$ (s, 2H), 7.85 $(\mathrm{d}, J=8.5 \mathrm{~Hz}, 4 \mathrm{H}), 7.48(\mathrm{t}, J=7.8 \mathrm{~Hz}, 2 \mathrm{H}), 7.32(\mathrm{t}, J=7.4 \mathrm{~Hz}, 1 \mathrm{H})$, $7.22(\mathrm{~d}, J=7.8 \mathrm{~Hz}, 2 \mathrm{H}), 7.18(\mathrm{~d}, J=8.5 \mathrm{~Hz}, 4 \mathrm{H})$.

Synthesis of $N$-phenyl-4-(1,4,5-triphenyl-1 $H$-imidazol-2-yl)- $N$ (4-(1,4,5-triphenyl-1H-imidazol-2-yl)phenyl)aniline (TPDBM). A mixture of 4,4'-(phenylazanediyl)dibenzaldehyde $(0.5 \mathrm{~g}, 1.65 \mathrm{mmol})$, aniline $(1.5 \mathrm{~g}, 16 \mathrm{mmol})$, benzil $(0.87 \mathrm{~g}, 4.1 \mathrm{mmol})$, ammonium acetate $(1.27 \mathrm{~g}, 16 \mathrm{mmol})$ and acetic acid $(30 \mathrm{ml})$ was refluxed under a nitrogen atmosphere in an oil bath for $24 \mathrm{~h}$. After cooling to room temperature, $200 \mathrm{ml}$ of water was added into the mixture. Then, the solid product was filtered out and washed with deionized water several times. Finally, it was further purified via column chromatography using dichloromethane/ethyl acetate (80/3) as the eluent to afford a white solid powder $(0.84 \mathrm{~g}, 60 \%) .{ }^{1} \mathrm{H}$ NMR $(500 \mathrm{MHz}$, $\left.\mathrm{CD}_{2} \mathrm{Cl}_{2}, \mathrm{ppm}\right): \delta=7.60(\mathrm{~d}, J=6.3 \mathrm{~Hz}, 4 \mathrm{H}), 6.90(\mathrm{~d}, J=8.8 \mathrm{~Hz}, 1 \mathrm{H})$, 7.46-7.23 (m, 24H), 7.22-7.17 (m, 4H), 7.17-7.10 (m, 7H), $6.95(\mathrm{~d}, J=8.5 \mathrm{~Hz}, 4 \mathrm{H})$. HRMS (mass, $m / z): 836.3\left[\mathrm{M}^{+}\right]$, $\mathrm{C}_{60} \mathrm{H}_{43} \mathrm{~N}_{5}$ calc.: 834.04.

Preparation of TPBMZ and TPDYZ. TPDBM $(0.5 \mathrm{~g}, 0.6 \mathrm{mmol})$ was dissolved in toluene and irradiated via a $365 \mathrm{~nm} \mathrm{UV} \mathrm{lamp}$ for $24 \mathrm{~h}$. After rotary evaporation, the resulting solution was purified via column chromatography using petroleum ether/ ethyl acetate (3/1) as the eluent. Two forms of yellow solid powder, TPBMZ (200 mg, 40\%) and TPDYZ (100 mg, 20\%), were obtained simultaneously. TPBMZ: ${ }^{1} \mathrm{H}$ NMR (500 MHz, DMSO, ppm): $\delta=7.63(\mathrm{~d}, J=8.8 \mathrm{~Hz}, 2 \mathrm{H}), 7.60(\mathrm{~d}, J=7.1 \mathrm{~Hz}, 2 \mathrm{H}), 7.57$ 
$(\mathrm{d}, J=7.0 \mathrm{~Hz}, 2 \mathrm{H}), 7.50(\mathrm{~d}, J=4.4 \mathrm{~Hz}, 1 \mathrm{H}), 7.49(\mathrm{~d}, J=7.1 \mathrm{~Hz}, 2 \mathrm{H})$, $7.43-7.15(\mathrm{~m}, 27 \mathrm{H}), 7.08(\mathrm{t}, J=7.2 \mathrm{~Hz}, 1 \mathrm{H}), 7.05$ (d, $J=7.5 \mathrm{~Hz}$, $2 \mathrm{H}), 6.88$ (d, $J=8.8 \mathrm{~Hz}, 2 \mathrm{H}), 6.78$ (d, $J=8.9 \mathrm{~Hz}, 2 \mathrm{H})$. HRMS (mass, $m / z$ ): $867.9\left[\mathrm{M}^{+}\right], \mathrm{C}_{60} \mathrm{H}_{43} \mathrm{~N}_{5} \mathrm{O}_{2}$ calc.: 866.04.

TPDYZ: ${ }^{1} \mathrm{H}$ NMR (500 MHz, DMSO, ppm): $\delta=7.67$ $(\mathrm{d}, J=8.8 \mathrm{~Hz}, 4 \mathrm{H}), 7.60(\mathrm{dd}, J=9.6,8.3 \mathrm{~Hz}, 8 \mathrm{H}), 7.51$ (t, $J=7.4 \mathrm{~Hz}, 2 \mathrm{H}), 7.41(\mathrm{dd}, J=15.0,7.4 \mathrm{~Hz}, 4 \mathrm{H}), 7.32(\mathrm{~m}, 12 \mathrm{H})$, $7.26-7.20(\mathrm{~m}, 5 \mathrm{H}), 7.09$ (t, $J=7.4 \mathrm{~Hz}, 2 \mathrm{H}), 7.00(\mathrm{~d}, J=7.3 \mathrm{~Hz}, 2 \mathrm{H})$, $6.83(\mathrm{~d}, J=8.8 \mathrm{~Hz}, 4 \mathrm{H})$.

Preparation of PMMA film doped with TPDBM. PMMA powder $(0.3 \mathrm{~g})$ was dissolved in acetone $(30 \mathrm{ml})$ followed by the addition of TPDBM or TPBMZ $\left(10^{-4} \mathbf{M}\right)$ in acetone solution. The mixed solution was heated at $60{ }^{\circ} \mathrm{C}$ for 30 minutes and then decanted onto a Petri dish, which was totally dried in a drying oven at $60{ }^{\circ} \mathrm{C}$ to obtain the doped film.

\section{Conclusions}

In summary, a new triphenylamine-imidazole molecule TPDBM has been designed and successfully synthesized, which exhibited an obviously large red-shifted aggregation-state emission peak at around $520 \mathrm{~nm}$. This unusual red-shifted emission was finally proved to arise from emission from a small number of products produced by a photochemical reaction involving TPDBM through an efficient Förster resonance energy transfer process, rather than from excimer emission. In addition, both of the corresponding photochemical products TPBMZ and TPDYZ exhibited excellent AIE properties, and TPBMZ could be further turned to TPDYZ due to the quite fast reaction rate. Furthermore, the photochemical reaction of TPDBM doped in an operable and hypotoxic PMMA film was employed as a sensitive fluorescent probe for oxygen with a lowest detectable oxygen volume ratio of $0.1 \%$. In this work, the actual origin of the newly apparent unusual red-shifted aggregation-state emission from an imidazole molecule has been confirmed, and this will make a contribution to basic photochemical reaction studies of imidazole derivatives. In addition, the introduction of this molecular configuration can also result in new material systems for sensitive fluorescent probes for oxygen.

\section{Conflicts of interest}

There are no conflicts to declare.

\section{Acknowledgements}

We are grateful for support from the National Natural Science Foundation of China (51603185, 51673174), and Zhejiang Provincial Natural Science Foundation of China (LY19E030006, LQ19E030016, and LZ17E030001).

\section{Notes and references}

1 M. G. Rabbani and H. M. El-Kaderi, Template-Free Synthesis of a Highly Porous Benzimidazole-Linked Polymer for $\mathrm{CO}_{2}$ Capture and $\mathrm{H}_{2}$ Storage, Chem. Mater., 2011, 23, 1650-1653.
2 Z. Li, Z. Cheng, J. Lin, N. Xie, C. Li, G. Yang and Y. Wang, Isomer dependent molecular packing and carrier mobility of N-phenylcarbazole-phenanthro[9,10- $d]$ imidazole based materials as hosts for efficient electrophosphorescence devices, J. Mater. Chem. C, 2019, 7, 13486-13492.

3 Y. Cui, Y. Zhao, T. Wang and B. Han, Benzimidazole-Linked Porous Polymers: Synthesis and Gas Sorption Properties, Chin. J. Chem., 2015, 33, 131-136.

4 Y. Xu, X. Liang, X. Zhou, P. Yuan, J. Zhou, C. Wang, B. Li, D. Hu, X. Qiao, X. Jiang, L. Liu, S. J. Su, D. Ma and Y. Ma, Highly Efficient Blue Fluorescent OLEDs Based on Upper Level Triplet-Singlet Intersystem Crossing, Adv. Mater., 2019, 31, 1807388.

5 Y. Dong, J. Qian, Y. Liu, N. Zhu, B. Xu, C.-L. Ho, W. Tian and W.-Y. Wong, Imidazole-containing cyanostilbene-based molecules with aggregation-induced emission characteristics: photophysical and electroluminescent properties, New J. Chem., 2019, 43, 1844-1850.

6 Y. Liu, T. Shan, L. Yao, Q. Bai, Y. Guo, J. Li, X. Han, W. Li, Z. Wang, B. Yang, P. Lu and Y. Ma, Isomers of PyreneImidazole Compounds: Synthesis and Configuration Effect on Optical Properties, Org. Lett., 2015, 17, 6138-6141.

7 B. Liu, Y. Yuan, D. He, D.-Y. Huang, C.-Y. Luo, Z.-L. Zhu, F. Lu, Q.-X. Tong and C.-S. Lee, High-Performance Blue OLEDs Based on Phenanthroimidazole Emitters via Substitutions at the C6- and C9-Positions for Improving Exciton Utilization, Chem. - Eur. J., 2016, 22, 12130-12137.

8 H. Liu, Q. Bai, L. Yao, H. Zhang, H. Xu, S. Zhang, W. Li, Y. Gao, J. Li, P. Lu, H. Wang, B. Yang and Y. Ma, Highly efficient near ultraviolet organic light emitting diode based on a meta-linked donor-acceptor molecule, Chem. Sci., 2015, 6, 3797-3804.

9 S. Xue, X. Qiu, S. Ying, Y. Lu, Y. Pan, Q. Sun, C. Gu and W. Yang, Highly Efficient Nondoped Near-Ultraviolet Electroluminescence with an External Quantum Efficiency Greater Than $6.5 \%$ Based on a Carbazole-Triazole Hybrid Molecule with High and Balanced Charge Mobility, Adv. Opt. Mater., 2017, 5, 1700747.

10 J. Shi, Q. Ding, L. Xu, X. Lv, Z. Liu, Q. Sun, Y. Pan, S. Xue and W. Yang, A simple D-p-A hybrid mode for highly efficient non-doped true blue OLEDs with $\mathrm{CIE}_{\mathrm{y}}<0.05$ and EQE up to 6\%, J. Mater. Chem. C, 2018, 6, 11063-11070.

11 W. C. Chen, B. Huang, S. F. Ni, Y. Xiong, A. L. Rogach, Y. Wan, D. Shen, Y. Yuan, J. X. Chen, M. F. Lo, C. Cao, Z. L. Zhu, Y. Wang, P. Wang, L. S. Liao and C. S. Lee, Deep-Red/ Near-Infrared Electroluminescence from Single-Component Charge-Transfer Complex via Thermally Activated Delayed Fluorescence Channel, Adv. Funct. Mater., 2019, 29, 1903112.

12 H. Zhang, J. Zeng, W. Luo, H. Wu, C. Zeng, K. Zhang, W. Feng, Z. Wang, Z. Zhao and B. Z. Tang, Synergistic tuning of the optical and electrical performance of AIEgens with a hybridized local and charge-transfer excited state, J. Mater. Chem. C, 2019, 7, 6359-6368.

13 K. Mutoh, N. Miyashita, K. Arai and J. Abe, Turn-On Mode Fluorescence Switch by Using Negative Photochromic Imidazole Dimer, J. Am. Chem. Soc., 2019, 141, 5650-5654. 
14 Y. Zhang, H. Li, G. Zhang, X. Xu, L. Kong, X. Tao, Y. Tian and J. Yang, Aggregation-induced emission enhancement and mechanofluorochromic properties of $\alpha$-cyanostilbene functionalized tetraphenyl imidazole derivatives, J. Mater. Chem. C, 2016, 4, 2971-2978.

15 Y. Zhang, J. Huang, L. Kong, Y. Tian and J. Yang, Two novel AIEE-active imidazole/ $\alpha$-cyanostilbene derivatives: photophysical properties, reversible fluorescence switching, and detection of explosives, CrystEngComm, 2018, 20, 1237-1244.

16 J. Wang, R. Li, X. Long and Z. Li, Synthesis of imidazolefunctionalized silicon quantum dots as "off-on" fluorescence probe for highly selective and sensitive detection of L-histidine, Sens. Actuators, B, 2016, 237, 740-748.

17 T. Gao, X. Cao, J. Dong, Y. Liu, W. Lv, C. Li, X. Feng and W. Zeng, A novel water soluble multifunctional fluorescent probe for highly sensitive and ultrafast detection of anionic surfactants and wash free imaging of Gram-positive bacteria strains, Dyes Pigm., 2017, 143, 436-443.

18 T. Gao, X. Cao, P. Ge, J. Dong, S. Yang, H. Xu, Y. Wu, F. Gao and W. Zeng, A self-assembled fluorescent organic nanoprobe and its application for sulfite detection in food samples and living systems, Org. Biomol. Chem., 2017, 15, 4375-4382.

19 R. Francke and R. D. Little, Optimizing Electron Transfer Mediators Based on Arylimidazoles by Ring Fusion: Synthesis, Electrochemistry, and Computational Analysis of 2-Aryl-1methylphenanthro[9,10- $d]$ imidazoles, J. Am. Chem. Soc., 2014, 136, 427-435.

20 H. C. Wu, A. D. Yu, W. Y. Lee, C. L. Liu and W. C. Chen, A poly(fluorene-thiophene) donor with a tethered phenanthro$[9,10-d]$ imidazole acceptor for flexible nonvolatile flash resistive memory devices, Chem. Commun., 2012, 48, 9135-9137.

21 D. Li, Q. Liu, J. Zhen, Z. Fang, X. Chen and S. Yang, ImidazoleFunctionalized Fullerene as a Vertically Phase-Separated Cathode Interfacial Layer of Inverted Ternary Polymer Solar Cells, ACS Appl. Mater. Interfaces, 2017, 9, 2720-2729.

22 Z. S. Wang, M. Li, Y. L. Peng, Z. Zhang, W. Chen and X. C. Huang, An Ultrastable Metal Azolate Framework with Binding Pockets for Optimal Carbon Dioxide Capture, Angew. Chem., Int. Ed., 2019, 58, 16071-16076.

23 Y. Ye, W. Guo, L. Wang, Z. Li, Z. Song, J. Chen, Z. Zhang, S. Xiang and B. Chen, Straightforward Loading of Imidazole Molecules into Metal-Organic Framework for High Proton Conduction, J. Am. Chem. Soc., 2017, 139, 15604-15607.

24 Y. Hu, N. Dunlap, S. Wan, S. Lu, S. Huang, I. Sellinger, M. Ortiz, Y. Jin, S. H. Lee and W. Zhang, Crystalline Lithium Imidazolate Covalent Organic Frameworks with High Li-Ion Conductivity, J. Am. Chem. Soc., 2019, 141, 7518-7525.

25 T. Fu, S. Smith, M. Camarasa-Gómez, X. Yu, J. Xue, C. Nuckolls, F. Evers, L. Venkataraman and S. Wei, Enhanced coupling through $\pi$-stacking in imidazole-based molecular junctions, Chem. Sci., 2019, 10, 9998-10002.

26 Q. Jiang, Y. Xu, X. Liang, C. Wang, X. Tang, Y. Li, X. Qiu, N. Zheng, R. Zhao, D. Zhao, D. Hu and Y. Ma, Stable HighEnergy Excited States Observed in a Conjugated Molecule with Hindered Internal Conversion Processes, J. Phys. Chem. C, 2019, 123, 6190-6196.
27 J. Liu, W. Li, M. Liu, Y. Dong, Y. Dai, Q. Song, J. Wang and C. Zhang, Photo-irradiated E/Z isomerization reaction of star-shaped isomers containing two cyanostilbene arms with charge transfer excited states, Phys. Chem. Chem. Phys., 2018, 20, 28279-28286.

28 W. Li, Y. Pan, R. Xiao, Q. Peng, S. Zhang, D. Ma, F. Li, F. Shen, Y. Wang, B. Yang and Y. Ma, Employing 100\% Excitons in OLEDs by Utilizing a Fluorescent Molecule with Hybridized Local and Charge-Transfer Excited State, Adv. Funct. Mater., 2014, 24, 1609-1614.

29 W. Li, Y. Pan, L. Yao, H. Liu, S. Zhang, C. Wang, F. Shen, P. Lu, B. Yang and Y. Ma, A Hybridized Local and ChargeTransfer Excited State for Highly Efficient Fluorescent OLEDs: Molecular Design, Spectral Character, and Full Exciton Utilization, Adv. Opt. Mater., 2014, 2, 892-901.

30 Y. Pan, W. Li, S. Zhang, L. Yao, C. Gu, H. Xu, B. Yang and Y. Ma, High Yields of Singlet Excitons in Organic Electroluminescence through Two Paths of Cold and Hot Excitons, Adv. Opt. Mater., 2014, 2, 510-515.

31 Q. Peng, W. Li, S. Zhang, P. Chen, F. Li and Y. Ma, Evidence of the Reverse Intersystem Crossing in Intra-Molecular Charge-Transfer Fluorescence-Based Organic Light-Emitting Devices Through Magneto-Electroluminescence Measurements, Adv. Opt. Mater., 2013, 1, 362-366.

32 S. Tang, W. Li, F. Shen, D. Liu, B. Yang and Y. Ma, Highly efficient deep-blue electroluminescence based on the triphenylamine-cored and peripheral blue emitters with segregative HOMO-LUMO characteristics, J. Mater. Chem., 2012, 22, 4401-4408.

33 S. Zhang, W. Li, L. Yao, Y. Pan, F. Shen, R. Xiao, B. Yang and Y. Ma, Enhanced proportion of radiative excitons in non-doped electro-fluorescence generated from an imidazole derivative with an orthogonal donor-acceptor structure, Chem. Commun., 2013, 49, 11302-11304.

34 S. Zhang, Y. Dai, S. Luo, Y. Gao, N. Gao, K. Wang, B. Zou, B. Yang and Y. Ma, Rehybridization of Nitrogen Atom Induced Photoluminescence Enhancement under Pressure Stimulation, Adv. Funct. Mater., 2017, 27, 1602276.

35 W. Li, D. Liu, F. Shen, D. Ma, Z. Wang, T. Feng, Y. Xu, B. Yang and Y. Ma, A Twisting Donor-Acceptor Molecule with an Intercrossed Excited State for Highly Efficient, Deep-Blue Electroluminescence, Adv. Funct. Mater., 2012, 22, 2797-2803.

36 B. S. Kim and J. Y. Lee, Engineering of Mixed Host for High External Quantum Efficiency above 25\% in Green Thermally Activated Delayed Fluorescence Device, Adv. Funct. Mater., 2014, 24, 3970-3977.

37 S. Zhang, L. Yao, Q. Peng, W. Li, Y. Pan, R. Xiao, Y. Gao, C. Gu, Z. Wang, P. Lu, F. Li, S. Su, B. Yang and Y. Ma, Achieving a Significantly Increased Efficiency in Nondoped Pure Blue Fluorescent OLED: A Quasi-Equivalent Hybridized Excited State, Adv. Funct. Mater., 2015, 25, 1755-1762.

38 W. Li, L. Yao, H. Liu, Z. Wang, S. Zhang, R. Xiao, H. Zhang, P. Lu, B. Yang and Y. Ma, Highly efficient deep-blue OLED with an extraordinarily narrow FHWM of $35 \mathrm{~nm}$ and a $y$ coordinate $<0.05$ based on a fully twisting donor-acceptor molecule, J. Mater. Chem. C, 2014, 2, 4733-4736. 
39 X. Du, B. Liu, L. Li, X. Kong, C. Zheng, H. Lin, Q. Tong, S. Tao and $\mathrm{X}$. Zhang, Excimer emission induced intra-system selfabsorption enhancement - a novel strategy to realize high efficiency and excellent stability ternary organic solar cells processed in green solvents, J. Mater. Chem. A, 2018, 6, 23840-23855.

40 Y. Yu, R. Zhao, C. Zhou, X. Sun, S. Wang, Y. Gao, W. Li, P. Lu, B. Yang and C. Zhang, Highly efficient luminescent benzoylimino derivative and fluorescent probe from a photochemical reaction of imidazole as an oxygen sensor, Chem. Commun., 2019, 55, 977-980.

41 A. Kundu, S. Karthikeyan, Y. Sagara, D. Moon and S. P. Anthony, Unusual fluorescent photoswitching of imidazole derivatives: the role of molecular conformation and twist angle controlled organic solid state fluorescence, Phys. Chem. Chem. Phys., 2018, 20, 27385-27393.

42 W. Yang, C. Li, M. Zhang, W. Zhou, R. Xue, H. Liu and Y. Li, Aggregation-induced emission and intermolecular charge transfer effect in triphenylamine fluorophores containing diphenylhydrazone structures, Phys. Chem. Chem. Phys., 2016, 18, 28052-28060.

43 J. Kai, M. C. F. C. Felinto, L. A. O. Nunes, O. L. Malta and H. F. Brito, Intermolecular energy transfer and photostability of luminescence-tuneable multicolour PMMA films doped with lanthanide- $\beta$-diketonate complexes, J. Mater. Chem., 2011, 21, 3796. 